\title{
Oriented Axon Projections in Primary Visual Cortex of the Monkey
}

\author{
Lawrence C. Sincich and Gary G. Blasdel \\ Program in Neuroscience and Department of Neurobiology, Harvard Medical School, Boston, Massachusetts 02115
}

\begin{abstract}
One important aspect of the functional architecture of primary visual cortex is the circuitry that accounts for the receptive field properties of neurons. The anatomy that underlies retinotopy and ocular dominance is well known, but no anatomical structure related to orientation selectivity has been found in primates. We examined whether the arrangement of local axon systems projecting within the cortical layers might be correlated with orientation preference in New World monkeys. We found that axons in layer 3 spread out from the site of a tracer injection in an anisotropic manner and that this elongated distribution is
\end{abstract}

Neurons in the primary visual cortex (V1) of macaque monkeys respond most briskly to a bar or grating stimulus when it is shown in the right part of visual space, to the appropriate eye, and within a small range of orientations. The anatomical substrates for the first two properties (retinotopy and ocular dominance) are the thalamic inputs to layer 4 , whereas selectivity for orientation has no established anatomical basis in primates. Unlike cat V1, where it appears that patterned input from the lateral geniculate nucleus to layer 4 provides the basis for orientation selectivity (Hubel and Wiesel, 1962; Ferster and Miller, 2000), neurons in layer 4C of primate $\mathrm{V} 1$ are insensitive to stimulus orientation. Instead, sharp orientation tuning is found in layers $2 / 3$ and $4 \mathrm{~B}$, one synapse removed from the thalamic input (Hubel and Wiesel, 1968; Dow, 1974; Bullier and Henry, 1980; Blasdel and Fitzpatrick, 1984; Anderson et al., 1993; Ringach et al., 1997). Consequently, orientation selectivity in primates seems to arise from intracortical circuitry. The neurons in layer $4 \mathrm{C}$ may provide patterned input to the superficial layers in a manner analogous to the thalamic input to layer 4 in the cat, but this idea has never been examined in primates. Another source for patterned input related to orientation tuning is the extensive lateral circuitry found in layers $2 / 3$ and 4B (Rockland and Lund, 1983). These intralaminar projections could capture the spatial geometry of a line stimulus by recapitulating retinotopy, specifically by distributing axon terminals along retinotopic axes.

Clear evidence of just such an arrangement has been discovered in tree shrew visual cortex, where layer $2 / 3$ neurons were found to project farther along cortical axes representing orientations that they prefer (Bosking et al., 1997). This warranted a search for similar anatomy in primates. However, a comparable finding would be difficult to demonstrate in macaques because it

\footnotetext{
Received Dec. 7, 2000; revised Feb. 20, 2001; accepted March 13, 2001.

This work was supported by the National Eye Institute and the Human Frontiers Science Program. We thank Drs. Daniel Adams, Jonathan Horton, David Hubel, and Niall McLoughlin and two anonymous reviewers for their helpful comments during the progress of this manuscript.

Correspondence should be addressed to Dr. Lawrence C. Sincich, Beckman Vision Center, University of California, San Francisco, 10 Kirkham Street, San Francisco, CA 94143-0730. E-mail: sincich@itsa.ucsf.edu.

Copyright (C) 2001 Society for Neuroscience $0270-6474 / 01 / 214416-11 \$ 15.00 / 0$
}

aligned with the preferred orientation recorded at each site. Moreover, within a few degrees of the foveal representation, the majority of the axon terminals fall within or just outside of the limits of the cortical mapping of the classical receptive field. Thus local axons produce a field of monosynaptic excitation that aligns with orientation axes and reaches neurons that have receptive fields which are adjacent in visual space.

Key words: visual cortex; New World primate; axon projections; orientation selectivity; retinotopy; receptive field would have to be embedded within the anisotropy of the visual field representation caused by ocular dominance columns (Van Essen et al., 1984; Tootell et al., 1988; Yoshioka et al., 1996; Blasdel and Campbell, 2001). To avoid this problem, we chose squirrel monkeys and owl monkeys for our experiments. The cortex of these New World monkeys exhibits weak anatomical segregation by eye (Kaas et al., 1976; Hendrickson et al., 1978; Horton and Hocking, 1996; O'Keefe et al., 1998). The lack of eye dominance in the upper layers is especially dramatic in squirrel monkeys, in which monocular tracer injections label all cytochrome oxidase blobs rather than just those in the ocular dominance columns of the injected eye, as in macaques (Horton and Hocking, 1996). Previously determined retinotopic maps in New World monkeys were too coarse for us to use, especially near the fovea where we recorded, so we used optical imaging techniques to generate cortical maps that represent the axes of visual space.

In examining the distribution of axon projections in layer 3 of New World monkeys, we found a close correspondence between the major axis of axon spread and orientation preference, quite similar to the finding in tree shrew by Bosking et al. (1997). The total reach of these projections corresponds to approximately three times the receptive field dimensions, which limits the retinotopic extent of monosynaptic influences to neurons representing adjacent receptive fields. Lateral projections and their patchy terminal distributions have been shown previously to be correlated with ocular dominance columns (Malach et al., 1993; Yoshioka et al., 1996), iso-orientation columns (Gilbert and Wiesel, 1989; Weliky et al., 1995), and cytochrome oxidase blobs (Livingstone and Hubel, 1984; Yabuta and Callaway, 1998b). Here we show that they also respect the axial constraint of retinotopy. Such axon projections form an intracortical circuit with the appropriate geometry and distribution to play a role in tuning neural responses to oriented stimuli.

\section{MATERIALS AND METHODS}

Animals. We used two species of New World primate: five adult squirrel monkeys (Saimiri sciureus) and two adult owl monkeys (Aotus trivirgatus). Monkeys were housed and cared for according to guidelines established by the National Institutes of Health and the Society for Neuroscience. 
Surgical preparation. Our surgical procedures are detailed in Blasdel (1992) and only briefly reviewed here. Twelve hours before each experiment, the animal was fasted and given a prophylactic dose of dexamethasone $(4 \mathrm{mg} / \mathrm{kg})$. Anesthesia was induced with ketamine supplemented by xylazine, during which we placed the animal in a stereotaxic frame, inserted an endotracheal tube, and provided inhalation anesthesia $(0.75-$ $1.0 \%$ isoflurane in a $50 \% \quad \mathrm{~N}_{2} \mathrm{O}-50 \% \quad \mathrm{O}_{2}$ mixture) for the remaining surgical procedures. We installed hard, gas-permeable contact lenses with $0.5 \%$ proparacaine and Neodecadron to protect the corneas.

Heart rate, end-tidal $\mathrm{CO}_{2}$, and temperature were monitored at all times and maintained at appropriate physiological levels. During recording episodes when the animal was paralyzed, we supported respiration with a respirator that had been modified to be under computer control. A catheter inserted into the cephalic vein provided an intravenous maintenance solution and supplemental thiopental anesthesia as needed $(5 \mathrm{mg} / \mathrm{ml})$. Our maintenance solution was drawn from a $500 \mathrm{ml}$ bag of Isolyte (Baxter) to which $20 \mathrm{mEq}$ of $\mathrm{KCl}, 1 \mathrm{gm}$ of ampicillin, and $1 \mathrm{gm}$ of chloramphenicol had been added and was infused at 4 $\mathrm{ml} \cdot \mathrm{kg}^{-1} \cdot \mathrm{hr}^{-1}$.

After we reflected the scalp from the posterior cranium, we made a 2 -cm-diameter craniotomy over the occipital pole of one hemisphere and then glued a stainless steel chamber onto the bone that we sealed with dental acrylic. We took care to insure that the dura was undisturbed and kept moist throughout the chamber implantation. A duratomy exposed most of the cortex inside the chamber, which included the V1 region representing the fovea and several degrees of the lower contralateral visual quadrant. We closed the chamber by threading into it a windowed insert formed by a stainless steel ring and a \#1-1/2 round glass coverslip. Closeable inlet and outlet tubes in the chamber allowed the intervening space to be filled with sterile balanced salt solution (BSS; Alcon, Ft. Worth, TX). This effectively damped any cortical movement caused by cardiac and respiratory pulsations and offered a mechanically stable preparation for recording.

Just before the physiological recording, we induced sufficient paralysis for ocular stability with tubocurarine (Curare; $0.6 \mathrm{mg} / \mathrm{kg}$, i.v.) and maintained it by continuous infusion with vecuronium bromide (4 $\left.\mathrm{mg} \cdot \mathrm{kg}^{-1} \cdot \mathrm{hr}^{-1}\right)$. We switched over to thiopental anesthesia $(0.5-1.0$ $\mathrm{mg} \cdot \mathrm{kg}^{-1} \cdot \mathrm{hr}^{-1}$, i.v.) and discontinued the isoflurane, respiring the animal with either $100 \%$ oxygen or room air during recording. We dilated the animal's pupils with atropine sulfate and adjusted the contact lenses to bring the eyes into focus on a screen either 1 or $2 \mathrm{~m}$ away, depending on the species.

Optical imaging. We used differential optical imaging to generate maps of orientation selectivity, ocular dominance, and retinotopy. In some animals, we used a voltage-sensitive dye (NK2367; Nippon KankohShikiso, Kenkyusho, Ltd., Okayama, Japan), and in other animals we imaged without dye. Details of the imaging technique are given in Blasdel (1992); here we present only those methods that have been adopted for these experiments.

Retinotopic maps have only recently been produced by optical imaging methods (Campbell and Blasdel, 1995; Bosking et al., 1997; McLoughlin and Blasdel, 1998). In these maps, vertical $\left(0^{\circ}\right)$, horizontal $\left(90^{\circ}\right)$, left $\left(45^{\circ}\right)$, and right $\left(135^{\circ}\right)$ obliquely oriented strips of the visual field are mapped on the surface of visual cortex. For each orientation, the stimulus consisted of our basic grating (four superimposed square-wave gratings of nonharmonic frequencies) moving behind a stationary occluding mask. Two masks were used to produce the difference image. Each mask consisted of parallel black bars of equal width and spacing, oriented the same way as the moving grating. The masks differed only in being spatial complements of each other. Subtracting the responses acquired during the presentation of each mask produced a map representing stripes of differentially activated cortex. Stimuli were presented monocularly and in most cases were produced for each eye to exclude effects caused by cyclotorsion. We varied the width and spacing of the bars of the mask to generate retinotopic maps at different spatial resolutions. Mapping with optical techniques is rapid as well as rigorous, because any eye movements cause a misregistration of the two stimuli that leads to degradation of the difference image.

The signal values for our optical images were centered around zero. To depict them in a 256-bit grayscale, we shifted zero to 127 and scaled the remaining values to span a comfortable middle region of this range for presentation purposes. We smoothed the images with a Gaussian kernel to remove high-frequency noise (for retinotopic maps, $\sigma=5$ pixels). Alignment of optical images with tissue sections was achieved by using a reference photograph of the surface vasculature and matching vessels that descended into the cortex to their profiles in the processed tissue [for details, see Yoshioka et al. (1996)].

Micropipette recording and receptive field mapping. Before making any micropipette recordings, we replaced the BSS under the chamber window with $2 \%$ agar in BSS to retain stability during recording. We used the same micropipette to make extracellular recordings and tracer microinjections so that there was no question of alignment. Borosilicate glass capillaries (1.2 mm diameter; Omnidot; World Precision Instruments, Sarasota, FL) were drawn on a pipette puller (David Kopf, Tujunga, $\mathrm{CA}$ ), and the tips were manually broken to a bevel while viewed at $200 \times$ magnification (5-7 $\mu \mathrm{m}$ inside diameter along the bevel). We front-filled the micropipette with a freshly made, saturated solution of biocytin ( $\geq 4 \%$; Sigma, St. Louis, MO) in sterile saline. We took a photograph of the cortical surface for later reference and chose injection sites in areas free of blood vessels and spaced $>3 \mathrm{~mm}$ apart. Just before introducing the pipette, we drilled a $\sim 0.5$-mm-diameter hole through the glass window with a flame-point carbide dental bit. We advanced the micropipette perpendicular to the pial surface to a depth of $500 \mu \mathrm{m}$ (estimated depth for layer 3 ) and recorded visually responsive single units or multiunits from a platinum-iridium wire inserted into the pipette. The pipette wire and a reference wire attached to the chamber were led to a differential amplifier (model P15; Grass Instruments, Quincy, MA), and the amplifier output was fed to a speaker so we could assess the neural activity. After we mapped the receptive field and made the injection at each site, the micropipette was removed, and a fresh one was used at the next location. All of the recordings were made in the dorsal occipital lobe, the region of $\mathrm{V} 1$ representing foveal and parafoveal vision $\left(0-4^{\circ}\right.$ eccentricity in squirrel monkeys and $0-10^{\circ}$ in owl monkeys).

At each site, the orientation preference and the range of orientations that elicited responses were judged by sound and recorded separately for each eye. We also mapped by hand the minimum response fields (Hubel and Wiesel, 1962). The borders and preferred orientation of the receptive field were determined as follows [adapted from Barlow et al. (1967)]: orientation was first established by sweeping a bar of white light $(\sim 1 \times$ $0.05^{\circ}$ ) over the receptive field and adjusting the orientation to produce a maximal response. We delimited the end borders by oscillating this oriented bar and moving it progressively along the axis of preferred orientation until the responses were eliminated. The lateral borders were delimited with the same oscillating bar, this time moving perpendicular to the orientation axis (on each side) until a clear response was no longer evoked. The stimulus length was then shortened to match the length of the receptive field, and the orientation was redetermined. The range of angles over which the cell was responsive was then plotted, and the midpoint of this range we designated the preferred orientation. We then replotted the receptive field borders using this optimal stimulus. Field sizes were in excellent agreement with previous reports in anesthetized (Hubel and Wiesel, 1968; Schiller et al., 1976a) and alert (Poggio, 1972; Dow et al., 1981; Snodderly and Gur, 1995) macaques. We did not find any published reports for receptive field sizes in New World monkeys, although O'Keefe et al. (1998) report a median optimal spatial frequency of 0.79 cycles $/{ }^{\circ}$ for their sample of complex cells in the owl monkey.

We made a total of $35 \mathrm{~V} 1$ injections in the seven animals (29 in squirrel monkeys and 6 in owl monkeys; any one hemisphere had 2-5 injections). In 14 of these injections we obtained successful recordings, cortical depth, and iontophoresis to yield data suitable for analysis.

Histology and reconstruction of biocytin labeling. After recording at each site, we passed 2-5 $\mu \mathrm{A}$ of anodal current (square wave; $7 \mathrm{sec}$ duty cycle) for $6-9 \mathrm{~min}$ to eject the biocytin iontophoretically. After all injections had been completed, we maintained each animal in an anesthetized state for at least $18 \mathrm{hr}$ before administering a lethal dose of pentobarbitol $(0.5 \mathrm{gm} / \mathrm{kg}$; Nembutal; Abbott Laboratories, North Chicago, IL). We perfused the animal with a warmed solution of $0.5 \%$ sodium nitrite in saline for $10 \mathrm{~min}$, followed by $4 \%$ paraformaldehyde (in $0.1 \mathrm{M}$ phosphate buffer) for $20 \mathrm{~min}$ and lastly $10 \%$ sucrose in phosphate buffer. We removed the occipital lobe and sank it in $30 \%$ sucrose at $4^{\circ} \mathrm{C}$ before histological processing.

The cortex was unfolded, flattened between two pieces of wax film, and quickly frozen against a copper block that had been chilled in dry ice. The tissue was fixed to a frozen stage with Tissue-Tek (Miles, Elkhart, IN) and then sectioned at $40 \mu \mathrm{m}$ intervals on a sliding microtome. We took care to preserve the first sections containing pial blood vessels that were vital for the subsequent alignment of processed tissue with functional maps derived from optical imaging. Three of every four tangential sections were reacted in separate wells for biocytin as follows [adapted from Horikawa and Armstrong (1988) and Adams (1992)]: sections were 
treated with (1) $1 \mathrm{ml}$ of $3 \%$ hydrogen peroxide, $10 \%$ methanol, and $0.1 \%$ sodium azide in $0.01 \mathrm{M}$ PBS to clear background for $45 \mathrm{~min}$; (2) $1 \mathrm{ml}$ of avidin-biotin complex solution (Vectastain Elite ABC; Vector Laboratories, Burlingame, CA) for $1 \mathrm{hr}$ on an agitator; (3) $1 \mathrm{ml}$ of PBS, $3 \mu \mathrm{l}$ of $3 \% \mathrm{H}_{2} \mathrm{O}_{2}$, and $1 \mu \mathrm{l}$ of biotinylated tyramine for $10 \mathrm{~min}$; (4) sections were returned to original wells of $\mathrm{ABC}$ solution (step 2) for $1 \mathrm{hr}$ and (5) $1 \mathrm{ml}$ of a diaminobenzidene (DAB) solution with heavy metal intensification consisting of $30 \mathrm{mg}$ of DAB (Aldrich, Milwaukee, WI), $60 \mathrm{ml}$ of $0.4 \mathrm{M}$ phosphate buffer, $90 \mu \mathrm{l}$ of $3 \% \mathrm{H}_{2} \mathrm{O}_{2}, 1.68 \mathrm{ml}$ of $1 \% \mathrm{CoCl}_{2}$, and $1.2 \mathrm{ml}$ of $1 \% \mathrm{Ni}\left(\mathrm{NH}_{4}\right) \mathrm{SO}_{4}$. We kept track of the background staining of the DAB reaction by inspection under a microscope. Each step was followed by three $5 \mathrm{~min}$ washes in PBS. The remaining sections were reacted for cytochrome oxidase (Wong-Riley, 1979) to verify the position of the border between V1 and V2. Except where specified, all reagents came from Sigma. Sections were dehydrated in ethanol followed by xylene before being coverslipped with a resin medium (Eukit, O. Kindler, Germany).

From sections corresponding to a depth of $300-600 \mu \mathrm{m}$ below the pial surface, we reconstructed axon fibers and terminals with a camera lucida at $100 \times$ magnification, while verifying axon terminals at $400 \times$. The high density of biocytin staining immediately around the injection site obscured the patch distribution; therefore nothing was reconstructed within a $200 \mu \mathrm{m}$ radius around the center of the injection. Because tissue flattening is generally imperfect, we analyzed sequential aligned sections for evidence of terminal projections and compiled the findings into one drawing. We used this drawing in all subsequent analyses of patch distributions. Photographic reconstructions were made using Adobe Photoshop (Adobe Systems, San Jose, CA) for illustrative purposes only.

Quantifying the axon terminal distribution. We based our delineation of axon terminal patches on the following criteria. (1) Terminal clusters must arise from the coalescence of more than one axon. This is a conservative threshold that eliminated stray fibers from the analysis but had the benefit of allowing us to focus on the patch system alone, to the exclusion of any radially diff use system of fibers. (2) When the density of staining was high in surrounding tissue (as it can be near the injection core), the identification of a patch had to be based on greater numbers of axon terminals, not on an accidental grouping of fibers passing through the area. (3) Patches should be of approximately uniform size for each injection, after taking into account the relative density of labeling. Taken together, these rules tended to underestimate the full extent of the lateral projections but allowed us to quantify a bona fide system of terminal patches.

To calculate whether axon terminal patches were distributed anisotropically around the injection site, we used a vector analysis to derive an index of ellipticity. The center of each patch (as determined by eye) was represented by a vector that projects from the center of the injection. An average vector, $R(r, \theta)$, was calculated for each injection site. The vector magnitude $r$ points from the injection site to the center of mass of all the patches and indicates the degree of ellipticity of each patchy system. The vector orientation $\theta$ may be compared directly with the orientation preference of units recorded from the pipette before injection. This orientation was calculated by doubling the angle of the individual patch vectors before adding, dividing the magnitude of this vector sum by the number of vectors, and dividing its orientation by two. This operation accounts for the fact that orientations are invariant for rotations through $180^{\circ}$ (for example, lines at 6 and $186^{\circ}$ are identically oriented). As a result, vector representations of patches located on opposite sides of an injection site, after their angles have been doubled, point in the same direction, whereas vector representations of patches diverging at $90^{\circ}$, after doubling, point in opposite directions, causing them to cancel if their magnitudes are the same. Patches located symmetrically around an injection site, for example, will yield a value of $r=0$, whereas patches distributed along an axis will produce a value of $r$ equal to the average distance of all patches from the injection site. Dividing $r$ by the mean length of all vectors used as input for the calculation, we arrive at a normalized index of ellipticity, $r_{n}$, with possible values ranging between 0 , indicating no ellipticity, and 1 , indicating a straight line.

The final calculation was to measure the difference between the average patch orientation $\theta$ and the angle of the retinotopic contour for the preferred orientation at the injection site. The value of $\theta$ was always made with respect to the anatomical boundary of the V1-V2 border; thus we only needed to know the angle of the retinotopic contour with respect to the border. This retinotopic angle was derived by drawing a tangent from the injection site on the appropriate retinotopic map to the V1-V2 border. However, because we generated maps of only four orientations $\left(0,45,90\right.$, and $\left.135^{\circ}\right)$, we interpolated intermediate values linearly. For instance, if we needed to estimate the retinotopic angle for a site preferring $22.5^{\circ}$, we used a value half way between the retinotopic angles provided by the 0 and $45^{\circ}$ retinotopic maps. The intercept angle of the resulting tangent line with the V1-V2 border was the value we compared against $\theta$ to determine whether lateral projections and local retinotopy were aligned.

\section{RESULTS}

\section{Anatomy of lateral projections by cortical layer}

Eleven of 14 biocytin microinjections we analyzed in layer 3 of New World monkey V1 revealed a highly stereotyped projection pattern of axons emanating from the injection site. Biocytin mainly filled the axons and dendrites of cells at the injection site, but a number of retrogradely labeled cells are also found in the tissue. Five examples from different squirrel monkeys are shown at low magnification in Figure 1, from sections cut parallel to the cortical surface. Several geometric features of the biocytin staining are apparent; anterogradely filled axons spread out radially and terminate in patches of denser staining, and these patches are distributed unevenly around the injection core. There is also a second set of projections that are diffuse and radially uniform around each injection. The remaining three microinjections we recovered were too small to produce sufficient biocytin transport such as that shown in Figure 1. Figure 2 is an injection made in owl monkey V1 with a similar pattern of asymmetric axon projections.

When the projection patterns are examined through the depth of the cortex, their lateral extent and geometry can be better appreciated, because they are distinctive between the cortical layers. Although the core of our injections was not always restricted to layer 3 (our target layer) and retrograde filling of cells can confound the projection patterns, the laminar origin of the lateral projections could be readily assessed by tracing fibers. Figure 3 shows sections of the upper right injection in Figure $1 B$ taken from different cortical depths. Beginning with layer 2, the staining consists almost solely of patchy, elongated projections and axon terminals. As layer 3 is approached, a diff use, shorterrange set of projections appears, superimposed on the patchy system that remains prominent in this layer. The diameter of the diffuse system is approximately equal to the diameter of the narrowest extent of the patch distribution. Continuing into layer 4B (Fig. 3C), the patches produced by the retrogradely filled cells in this layer are less pronounced, although they remain in register with those in the superficial layers, and the diffuse system is prevalent. In layer $4 \mathrm{~B}$ and in layers $4 \mathrm{C}$ and 5 , the number of retrogradely labeled cells increases, consistent with the dense columnar projections from these layers to layer 3 . It is worth noting that the labeled cells in layer 4B are situated along the same elongated axis as the axons. No patchy projections arise from cells in layer $4 \mathrm{C}$ (Fig. 3D,E), and the lateral projections remain diffuse but are considerably shorter. In layer $4 C \beta$, the staining appears almost exclusively as the cell bodies and dendrites of retrogradely labeled cells. These neurons form a small circle of cells $\sim 400 \mu \mathrm{m}$ in diameter, slightly larger than the injection core in layer 3. A composite plot of the labeled cells in layer $4 \mathrm{C} \beta$ revealed a circular distribution of cells, which was true for all our injections. Figure $3 F$ contains a small cluster of cells labeled in layer 5. These cells, as well as the efferent fibers descending from layers $2 / 3$, send out a large number of axon collaterals that radiate uniformly with little hint of patch structure.

A composite reconstruction of several adjacent sections in layer 

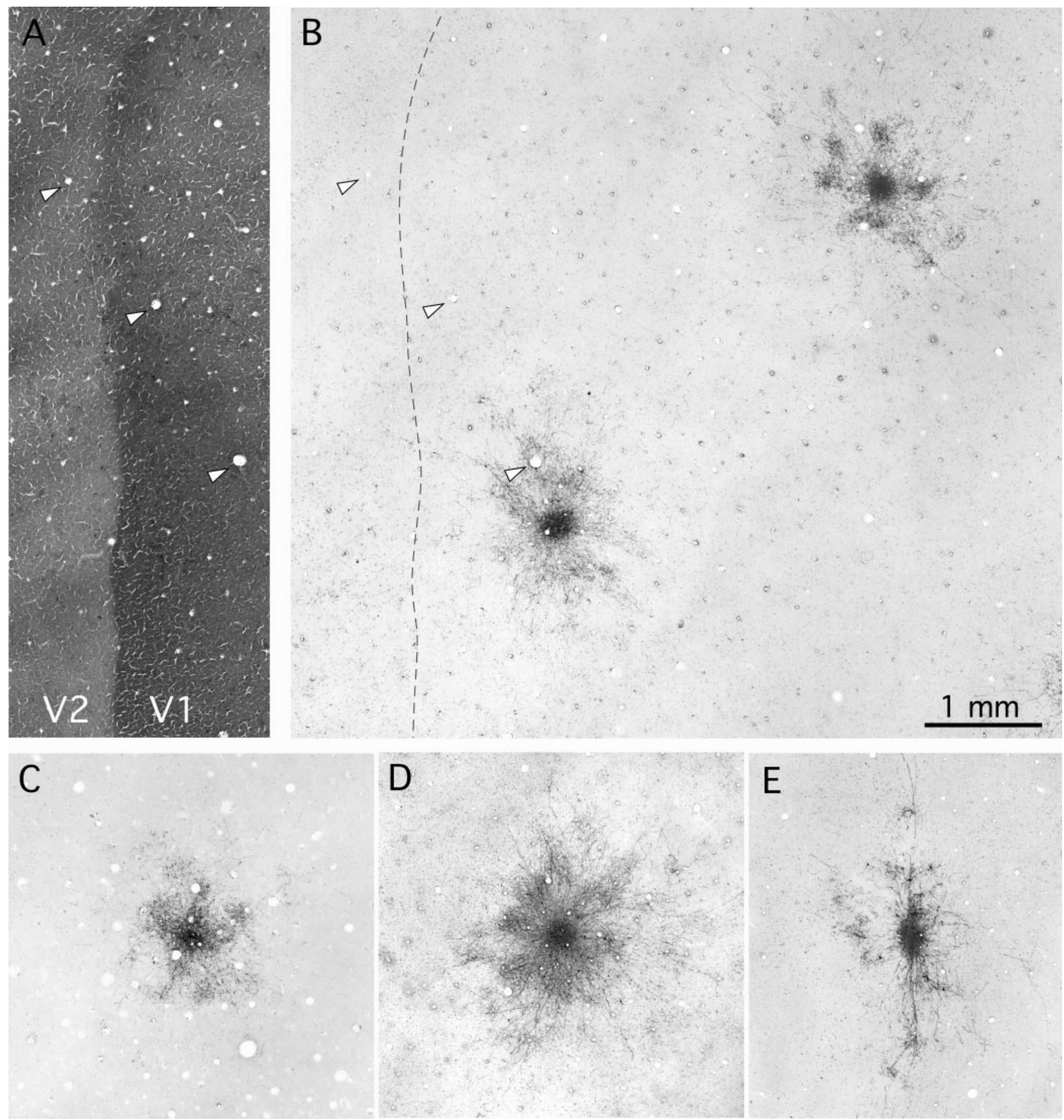

Figure 1. Elongated distribution of lateral axon projections in layer 3 revealed by microinjections of biocytin in squirrel monkey V1. Each image has been rotated and/or flipped to keep the V1-V2 border at the left and the foveal representation at the top. A, Cytochrome oxidase-stained section of flattened cortex showing V1-V2 border in layer 4. Arrowheads indicate blood vessels that are found in the layer 3 section shown in $B$. B, Photomontage of a single section of flattened cortex containing two microinjections of biocytin in layer 3. The foveal representation in V1 lies just beyond the top of the picture. The dashed line is the V1-V2 border as shown in $A$, running anteromedially from top to bottom. $C-E$, Photographs of single sections of injections from three other squirrel monkey hemispheres illustrating the range of patch distributions, from nearly circular $(C)$ to highly elongated $(E)$. Scale bar: $A-E, 1 \mathrm{~mm}$.

3 helped verify the presence of a patch of axon terminals, especially in sparse areas $>750 \mu \mathrm{m}$ from the injection site. We restricted our analysis of the patch system to layers $2 / 3$ by examining sections no deeper than $600 \mu \mathrm{m}$ below the pial surface. By photographically superimposing sections, a more complete account of the patches appears, which is apparent when comparing the injection in Figure 3 with its reconstruction at higher magnification in Figure $4 A$. At this scale, it is clear that squirrel monkey patches are $\sim 200 \mu \mathrm{m}$ in diameter and are separated by zones relatively free of terminals, especially when those patches are $>500 \mu \mathrm{m}$ from the injection center. This configuration is similar to the "lattice connections" described by Rockland and Lund 


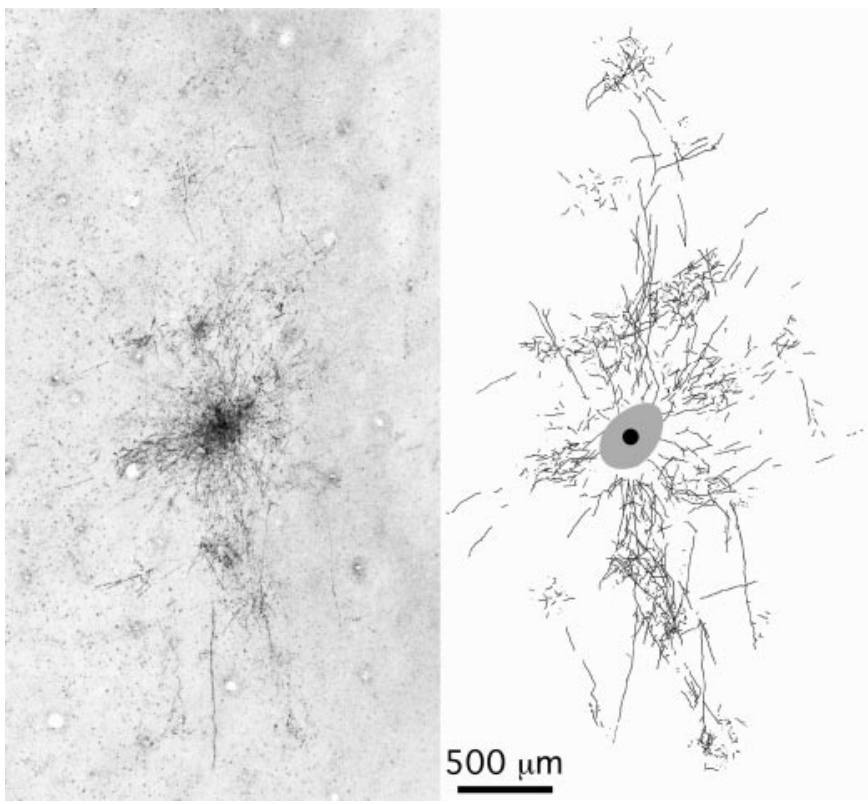

Figure 2. Surface view of lateral axon projections in layer 3 of owl monkey V1. Left, A photograph of a single section. Right, A camera lucida drawing compiled from several adjacent sections.

(1983), who used horseradish peroxidase as their tracer. It is also comparable with the patchy system of terminals found in layer 3 of macaque V1 (Yoshioka et al., 1996). In our sample of injections, patches could often be found $\sim 1 \mathrm{~mm}$ from the injection center but rarely farther than $1.5 \mathrm{~mm}$.

We simplified our analysis of the patch distribution by reducing the reconstructions to a set of circles indicating patch positions and the injection center. Patches were discriminated by noting where presynaptic terminals from more than one axon congregate, which at low magnification was manifested by denser labeling compared with the surrounding tissue (Fig. 4B). Patches buried within the radially diffuse labeling (Fig. $4 C$, large dashed circle) also became more apparent by superimposing sections. Each injection was eventually reduced to a set of circles representing the patches and the injection center (Fig. 4D). The lateral distribution of patches in each case filled a region of cortex that could be described by an ellipse, so we calculated a normalized index of ellipticity for each site using a vector notation (see Materials and Methods). The resulting vector orientation $\theta$ was the angle of the major axis of the ellipse, and its magnitude $r_{n}$ indicated the degree of anisotropy of the patch system. The elliptical distribution is apparent when the reconstructions are oriented along the same axis, as in Figure 5. The mean index of ellipticity for all injections was 0.42 , indicating that patches were typically 1.7 times farther from the center of the injection along the major axis of the ellipse than along the minor axis. In no case was a distribution found with an index $<0.2$.

\section{Lateral patchy projections in layer 3 are retinotopically aligned with orientation preference}

From a casual inspection of the injections in Figure 1, it appears that the long axes of these projections fall at different angles with respect to the V1-V2 border (which has been oriented vertically in the examples shown). Hence, in retinotopic terms, it is clear that the axes of elongation occur at angles representing different orientations in visual space. The V1-V2 border, as revealed by cytochrome oxidase staining, was used as a reference landmark to determine whether the patches systematically extended along a particular retinotopic axis. Because we used the biocytincontaining micropipette as a recording electrode before tracer injection, we were able to map the receptive field properties of neurons at every site. All of the neurons recorded were complex cells. Receptive field sizes were $<0.5^{\circ}$ in width and length for each eye, whereas the orientation tuning full-width varied from 42 to $125^{\circ}$, the midpoint of which was designated the preferred orientation. The aspect ratio of the receptive fields and the orientation tuning widths were found to be uncorrelated with the ellipticity of the patches $\left(r_{n}\right)$.

To determine the relationship between the orientation of the receptive field and the orientation of the lateral projections in layers $2 / 3$, we generated a set of retinotopic maps of the visual axes on the surface of V1 where we placed the injections. We produced a set of such maps with differential optical imaging and aligned them with the histological material using major blood vessels and the V1-V2 border as guides. The patterns we obtained from the monkeys shown in Figure 6 make it clear that representations of straight edges can curve radically (and along some visual axes, unpredictably) within a few millimeters of the V1-V2 border. If we had analyzed our injection data on the basis of the V1-V2 border alone, without reference to the retinotopic maps, we would have been seriously misled.

Alignment of an injection from a squirrel monkey is shown in Figure $6 A$, and one from an owl monkey is shown in Figure $6 B$. The projection patterns are superimposed over differential images of vertical and horizontal meridia from the same regions of cortex in which the injections were made. Drawings on the left show the size and orientation preference of the receptive fields of the neurons, along with their location in visual space. In both examples the axons formed terminal patches at greater distances along the retinotopic contours for the preferred orientation of each site (middle panels). This is emphasized by comparing the patch distributions with the retinotopic maps for the nonpreferred contours (right panels), where the patches appear spread out against the grain.

After injections could be superimposed onto retinotopic maps, it was straightforward to measure how closely aligned the long axis of the patch distribution was to the preferred orientation of the site. We found that the mean difference between the orientation of the receptive field and the orientation of the lateral projections was $12^{\circ}$ for 11 injections. This agreement is exemplified in Figure 7, where all the injection sites are shown schematically, each scaled by the magnification factor appropriate for its eccentricity and oriented with its receptive field in visual coordinates. Although statistical analyses are difficult to apply to several of the quantities we measured, such as patch distributions and retinotopic contours, it is remarkable how often the alignment is very close, within $10^{\circ}$ in 7 of 11 cases (Fig. 8). In cortical coordinates, therefore, upper layer pyramidal neurons project farthest along axes representing preferred contours in visual space. This finding provides the first evidence of a correlation between axonal geometry and orientation preference in a primate.

When the same alignments are done using retinotopic maps for squirrel monkeys provided in Cowey (1964) and for owl monkeys in Allman and Kaas (1971), the mean difference increased to $28^{\circ}$. This illustrates the importance of defining the retinotopic map in individual animals using optical imaging, a method that rapidly and reliably plots local retinotopic contours at high spatial resolution. 


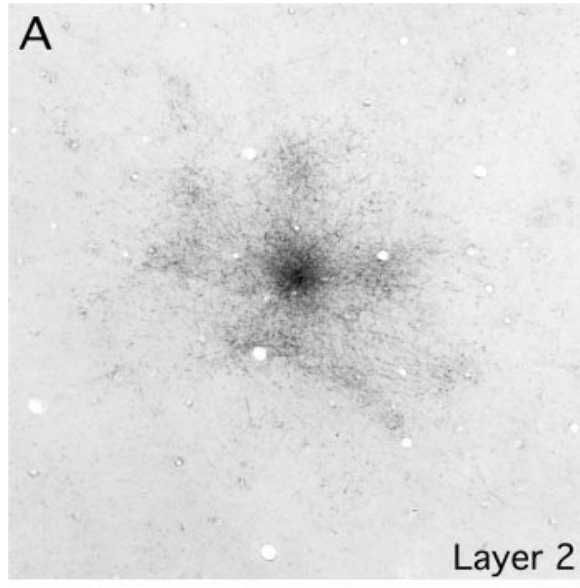

D

Layer $4 \mathrm{C} \alpha$
B

Layer 3

E

Layer $4 \mathrm{C} \beta$
C

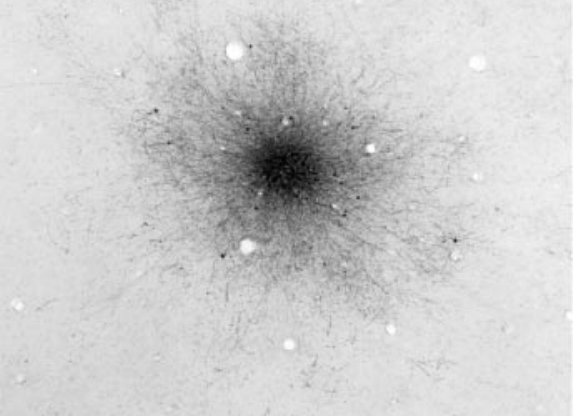

Layer 4B

F

$500 \mu \mathrm{m}$

Layer 5

Figure 3. Lateral axon projections terminate in a patchy, anisotropic manner predominantly in the supragranular layers. $A$, In the most superficial layer where biocytin label appeared (layer 2), the axon terminals formed an almost pure patch system. $B$, Deeper in layer 3 , where the microinjections were made, this patch system is partially obscured by the presence of shorter, radially diffuse axons. $C-E$, A patch system is still apparent in layer $4 \mathrm{~B}$ but disappears in layer $4 \mathrm{C}$, where a number of retrogradely filled neurons are clustered below the injection site. $F$, In layer 5 collaterals of efferent axons from cells in layer 3 and 5 make a radial starburst pattern with no sign of patchy terminations.

\section{Anisotropy of the lateral projections is not caused by other known factors}

The degree of anisotropy found in the patch distribution is mild compared with that found in the tree shrew (Bosking et al., 1997). It is possible that other factors may have produced it in New World monkeys. One possibility is that cortical magnification may vary with the visual axis, as it does in the macaque near the fovea (Van Essen et al., 1984; Tootell et al., 1988). However, direct measures of retinotopic representations in squirrel and owl monkeys reveal much less distortion, $\sim 1.1: 1$ versus 1.6:1 for macaques (Blasdel and Campbell, 2001), which is too small to explain the average anisotropy of 1.7:1 in these injections. Another possible source of anisotropy is the gradient in cortical magnification that runs along axes projecting radially from the fovea, in contrast to isoeccentricity axes along which cortical magnification should be constant. Because we found elongations correlating with all axes and observed no particular preference for those close to radial, we believe this can be excluded. Finally, anisotropy because of ocular dominance may still occur in the presence of weak functional segregation (Horton and Hocking, 1996), but the hint of ocular dominance occasionally observed by optical imaging (data not shown) had no obvious correlation to the elongated terminal distributions.

\section{Receptive field size compared with axon spread}

Because the retinotopic maps enabled us to calculate the value of the cortical magnification factor locally, we could also estimate the cortical dimensions of the receptive field and compare it with the spread of axon terminal patches. This comparison allowed us to determine whether the span of the projections matched the dimensions of the receptive field or whether it reached cortical territory representing the visual surround. For example, in Figure 7 , the top left injection, the cortical magnification factor for this site was $2.5 \mathrm{~mm} /{ }^{\circ}$, and the receptive field recorded at the site was $0.2 \times 0.2^{\circ}$. When the receptive field is scaled by this magnification value and superimposed over the terminal distribution, it covers only $0.5 \mathrm{~mm}$ of the $2.1 \mathrm{~mm}$ span of the lateral projections. The patches spread out beyond the limits of the receptive field, true for all of the injections, with the more distant patches found close to the axis defined by their orientation preference. All of the squirrel monkey injections were made within $2^{\circ}$ of the foveal representation, and their patch systems covered two to three times the diameter of the representation of the receptive field, far enough to synapse onto neurons with adjacent but nonoverlapping receptive fields. This point is evident when the patch systems for all of the squirrel monkey injections are superimposed and aligned along the axis of the orientation of their receptive fields, 

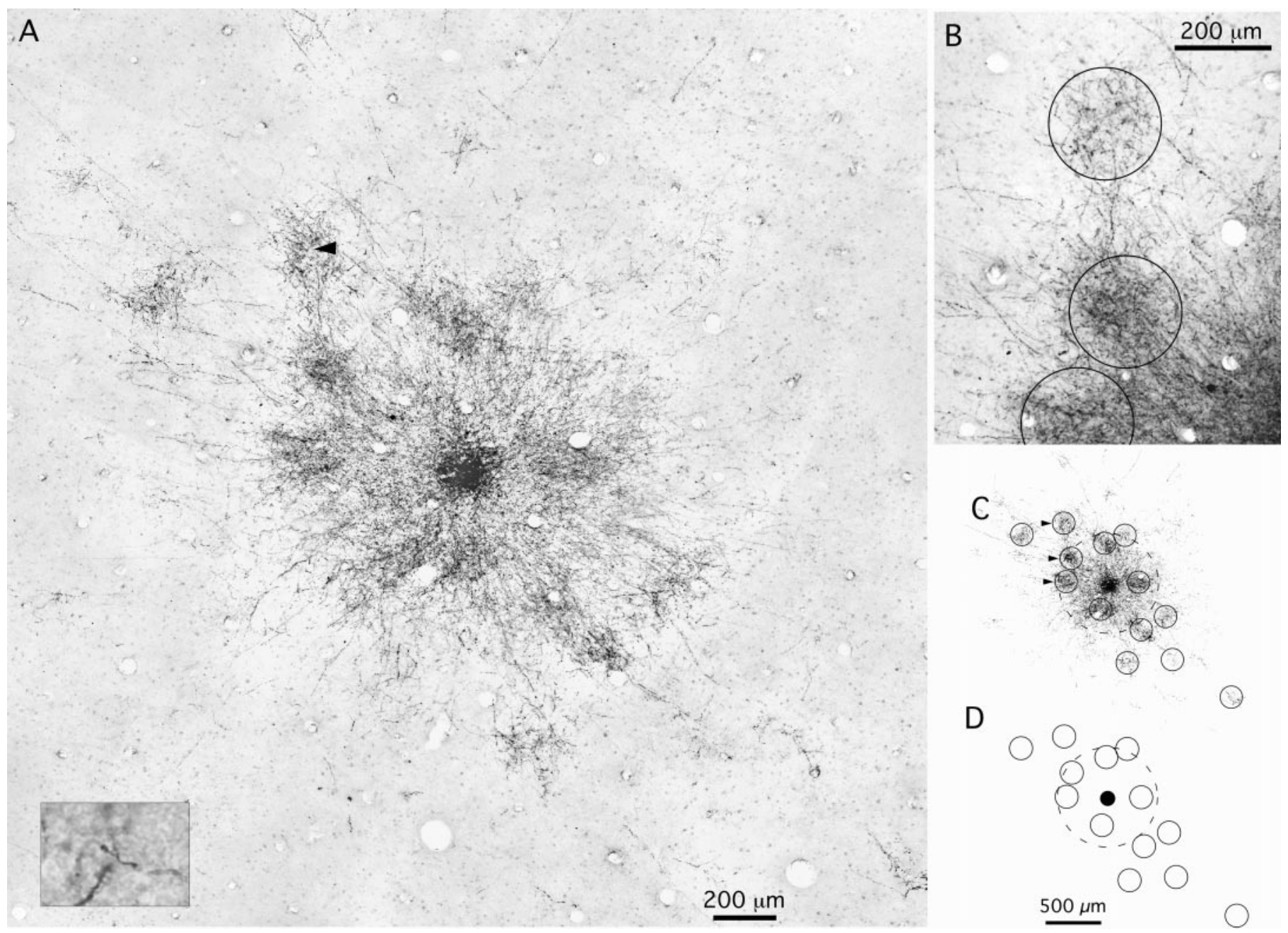

Figure 4. Determination of the axon terminal patch distribution through the depth of layers 2 and 3 . A, A photographic reconstruction of the patch system for the injection shown in the upper right corner of Figure $1 B$. A more complete picture of the patch system is made by collapsing the biocytin labeling from many sections onto one image. Inset, A pair of axon terminal boutons (located at the arrowhead in the reconstruction) photographed with a $100 \times$ objective. $B$, Detail of three patches (circles) from a single section of this injection, showing how patches can be plotted on the basis of the relative density of biocytin labeling. $C$, Reduction of the biocytin label to a set of circles representing the axon terminal patches. The three patches in $B$ are indicated by arrowheads in this thresholded image of the injection. The large dashed circle indicates the approximate extent of the diff use projections that do not terminate in a patchy style. $D$, Final plot used for analyzing the geometry of the patch system.

as in Figure $9 A$. When that ensemble is Gaussian smoothed (Fig. $9 B)$, it becomes apparent that the average receptive field size (drawn as a white rectangle) is approximately one-third the diameter of the lateral projections.

Although axons in layer 3 labeled with biocytin may be either excitatory or inhibitory, those traveling $>300 \mu \mathrm{m}$ from the injection site are most likely collaterals of excitatory pyramidal neurons, because in the macaque nonspiny, presumably GABAergic cells rarely project beyond this distance (Lund and Yoshioka, 1991; Lund and $\mathrm{Wu}, 1997)$. Of these axons, at least $80 \%$ of their synapses are made with other pyramidal neurons (McGuire et al., 1991; DeFelipe and Fariñas, 1992). The sum of these results suggests that the lateral projections in layer 3 produce a field of monosynaptic excitation that is aligned with orientation axes and can deliver input to neurons with adjacent, nonoverlapping receptive fields.

\section{DISCUSSION}

Since their discovery by Rockland and Lund (1983), the lateral patchy projections of layers $2 / 3$ have been related to a number of architectural features in primate V1. Livingstone and Hubel (1984) and Yabuta and Callaway (1998b) have shown that neurons terminate preferentially in areas with similar levels of cytochrome oxidase staining. Several groups have shown that lateral projections tend to connect regions with the same ocular dominance and with similar orientation preferences (Gilbert and Wiesel, 1989; Malach et al., 1993; Weliky et al., 1995; Yoshioka et al., 1996; Bosking et al., 1997). Most recently, it has been shown that these projections also align with retinotopic axes, according to their preferred orientation, in tree shrews and in cats (Bosking et al., 1997; Schmidt et al., 1997). We have extended this last finding to New World monkeys.

Previous work in macaque monkeys had shown that the patches of layer 3 neurons are oriented along a cortical axis that is functionally significant. Most often, the projections are elongated perpendicular to the ocular dominance columns (Amir et al., 1993; Yoshioka et al., 1996), although individual neurons will project parallel to the columns (McGuire et al., 1991). Near the V1-V2 border, where injections are commonly made, the ocular 


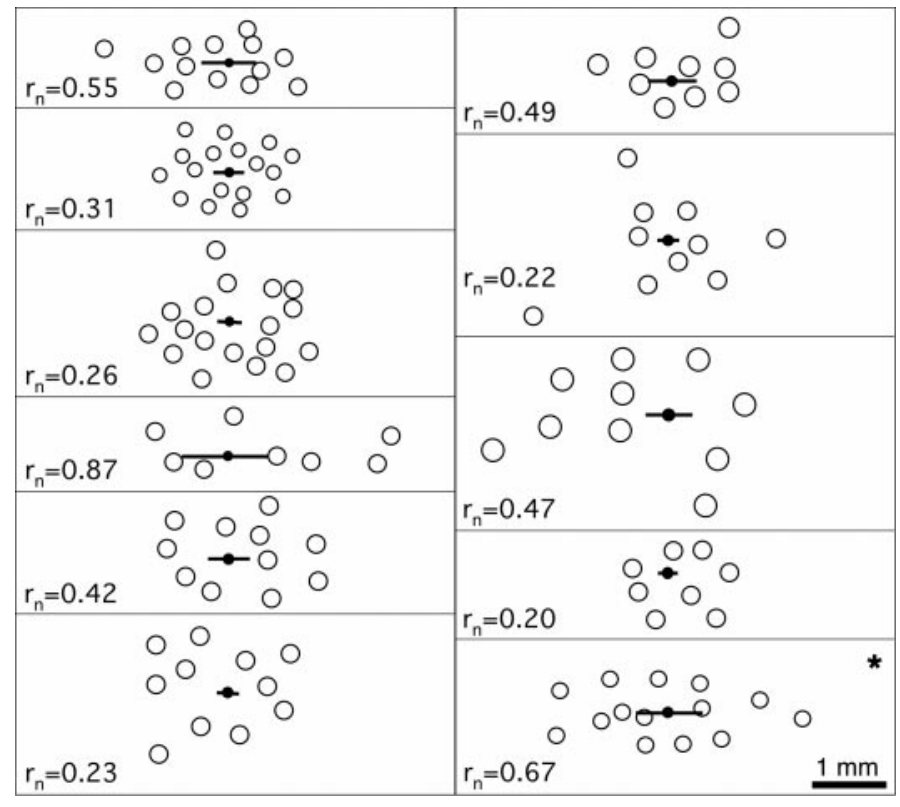

Figure 5. Patch distributions are not circularly symmetric. Patch plots (white circles) surrounding injection sites (black circles) illustrate how the plots are dispersed along one axis. The length of the heavy line is proportional to the normalized vector magnitude $\left(r_{n}\right)$ of the patch positions with respect to the center of the injection. The top left reconstruction is the injection shown in Figures 3 and 4. The asterisk indicates the reconstruction from the owl monkey injection in Figure 2. dominance columns usually form a regular stripe pattern. This pattern produces an anisotropy in the local cortical magnification factor of $\sim 1.6: 1$ [across versus with the stripes (Dow et al., 1985; Blasdel and Campbell, 2001)]. The elongated projections in macaques have a slightly larger aspect ratio [1.69:1 (Malach et al., 1993); 1.78:1 (Yoshioka et al., 1996)]. Because their long axes run in the direction of the anisotropy, it is unclear whether the projections are set down to maintain ocularity or to compensate for the unequal magnification of the visual field.

These issues obscure the possibility that projections may be related to orientation axes as well. However, the anisotropy in cortical magnification is not high everywhere in macaque V1. In regions where the ocular dominance pattern is irregular rather than stripe-like, the anisotropy can approach 1.1:1 (Blasdel and Campbell, 2001), similar to the values in New World monkeys. This raises the possibility that in animals in which ocular dominance columns are present, but the pattern does not produce an anisotropy in cortical magnification, elongated projections may still be correlated with orientation axes. It remains to be seen whether this is true in macaque cortex, especially near the horizontal meridian where the ocular dominance pattern is often less stripe-like.

Because lateral projections are aligned with orientation preferences, they could be related to receptive field properties in several ways. One possibility is that the degree of ellipticity in the projections $\left(r_{n}\right)$ is correlated with orientation tuning width. Because we found no correlation between $r_{n}$ and tuning width, it suggests there is a different aspect of the intracortical wiring that
A

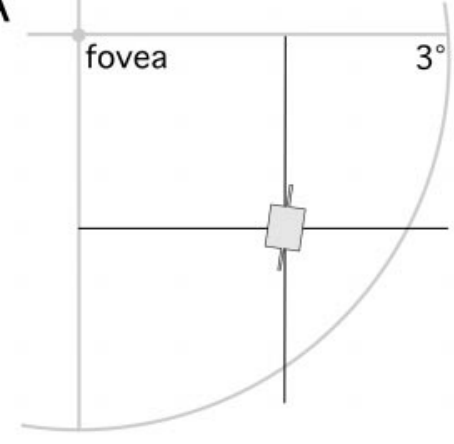

B

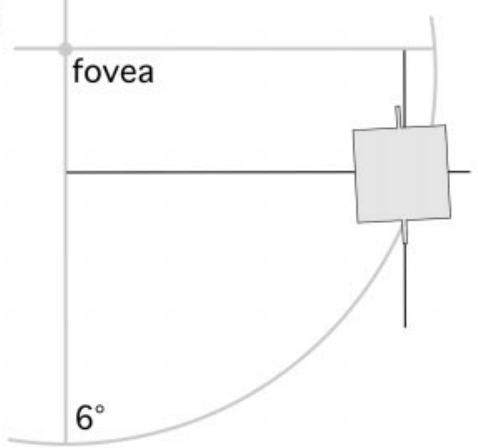

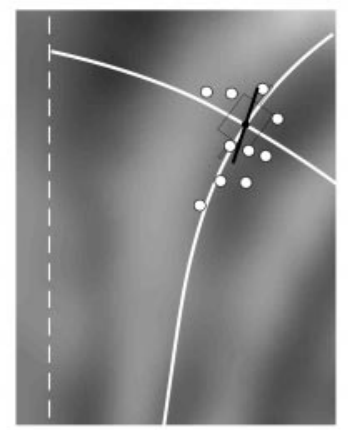
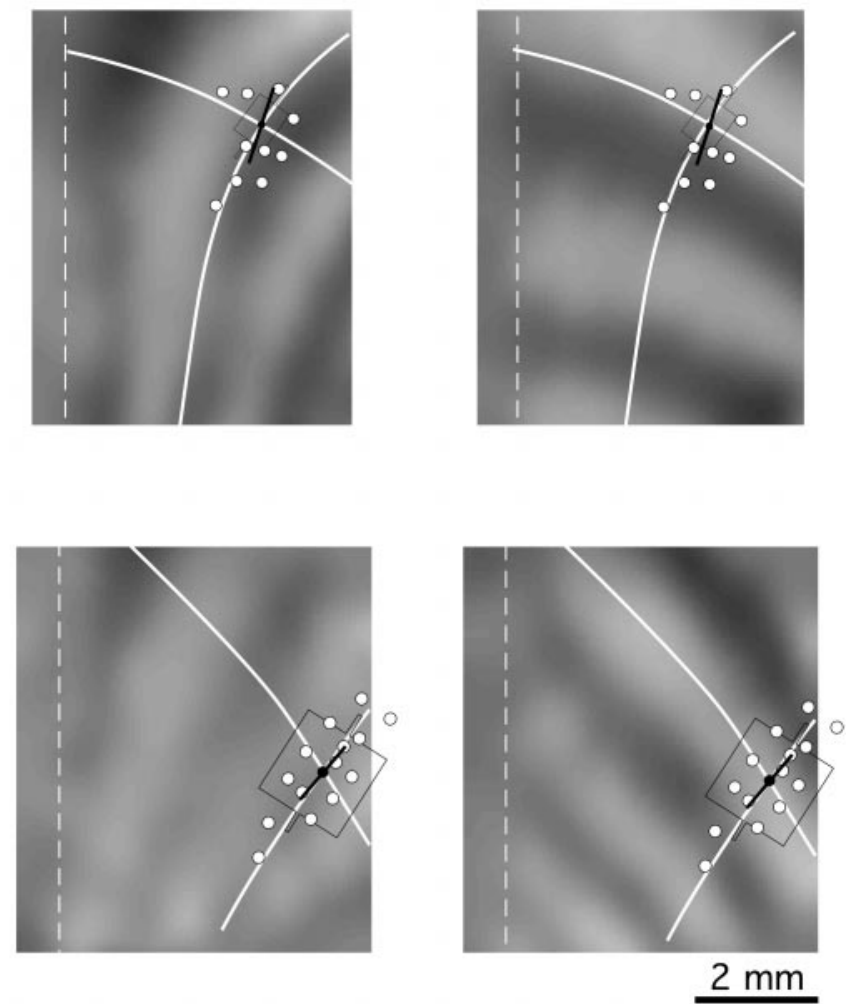

Figure 6. The long axis of the patchy axon projections aligns with the retinotopic axis of the preferred orientation recorded at the injection site. A, Alignment of one injection in a squirrel monkey. Left, The vertical-preferring receptive field and its coordinates recorded at the injection site. Middle, The retinotopic map for vertical lines, over which is drawn the patch system and the coordinate axes of the injection. The dashed line indicates the V1-V2 border. Right, For comparison, the same region of V1 with the retinotopic map for the nonpreferred orientation, in this case, horizontal lines. B, Alignment of a vertical-preferring injection site in an owl monkey. Panels are as described in $A$. Scale bar of all retinotopic images, 2 mm. 


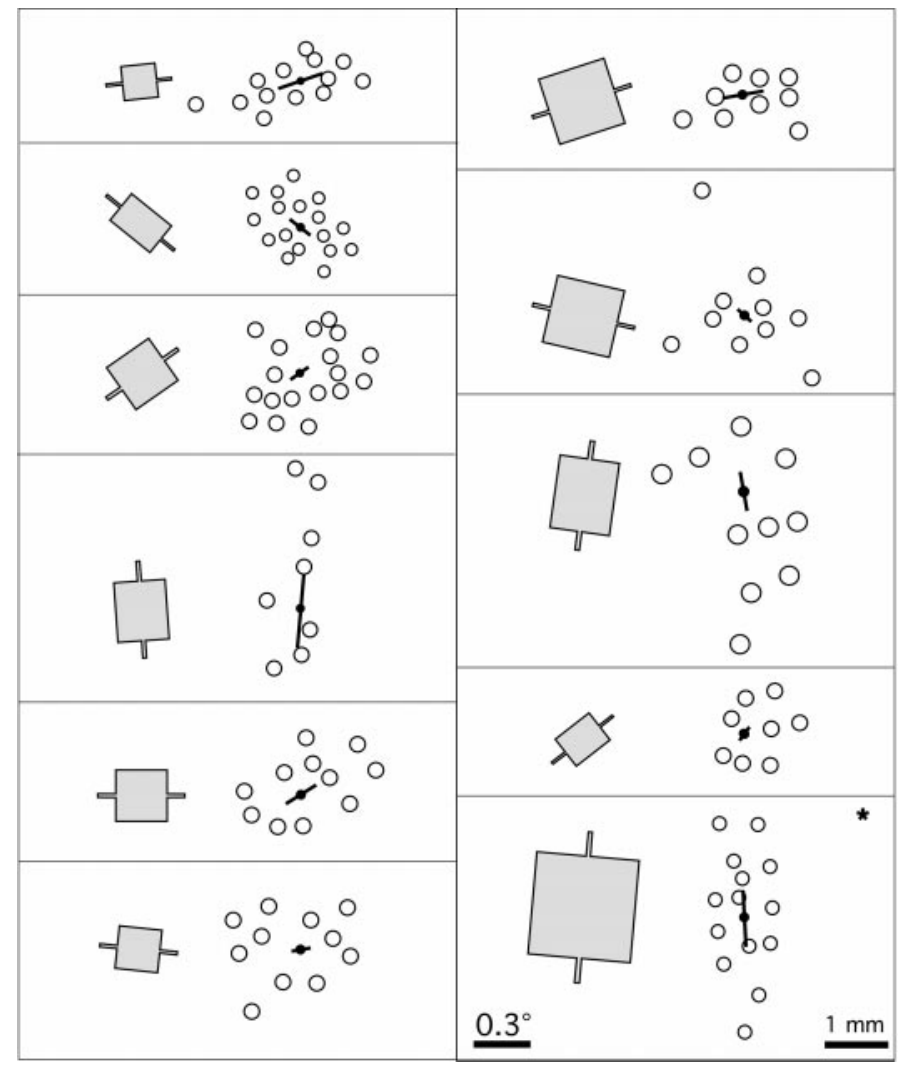

Figure 7. Patchy axon terminals are distributed along the axis corresponding to their preferred orientation. Side-by-side comparison of receptive fields (left) and patch distributions (right) of all the injections, oriented via retinotopic maps and scaled according to the local cortical magnification factor. The asterisk indicates the owl monkey injection in Figure 2.

determines this variable. For instance, are sharply tuned cells more likely to be mutually connected when they are positioned coaxially? The relatively weak ellipticity revealed by our injections makes such specific wiring a better candidate.

Receptive field size is another property that might be determined by the lateral projections. This hinges on how the receptive field is defined, and certainly the method we used to plot the minimum response field is not the only way to measure receptive fields (Skottun et al., 1991). We can only reiterate that the field sizes of the complex cells we recorded approximate the visual area over which a stimulus must pass for the neuron to fire. In addition, our patch reconstructions were conservative, underestimating the total span of the lateral projections. With this in mind, it appears that the projections do extend beyond the cortical dimensions of the receptive field representation, although not exceptionally far. Because the longest projections arise from excitatory neurons (Lund and Yoshioka, 1991; Lund and Wu, 1997), it seems unlikely that the projections directly determine the bounds of the minimum response field. It is possible that a combination of excitatory and inhibitory effects acting over the projection field might determine field size, but we have no way to assess their relative contributions. Input from layer $4 \mathrm{C}$ is certainly involved, and it is worth noting that the distribution of retrogradely labeled cells in this layer averaged $\sim 400 \mu \mathrm{m}$ in diameter, slightly smaller than the cortical dimensions of the receptive field. The combined input sources must determine field size in the end, and we should recall that receptive field boundaries are not

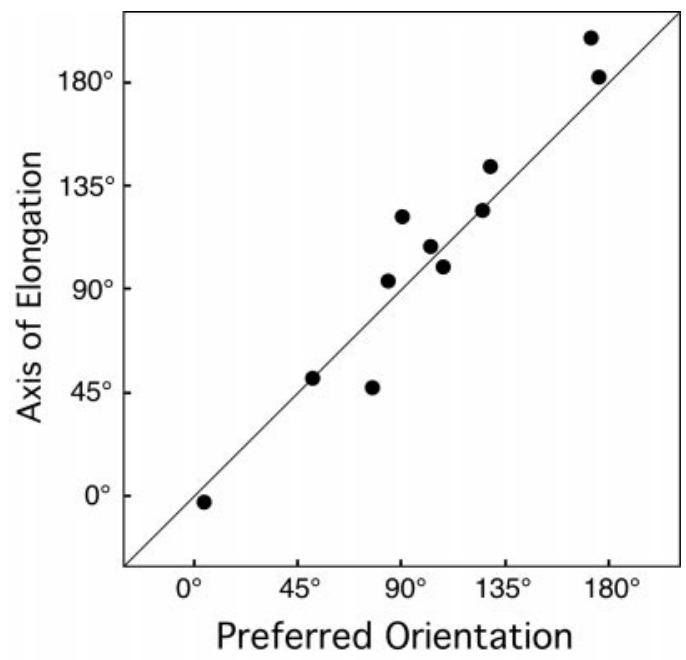

Figure 8. The preferred orientation is plotted against the orientation of the axis of elongation for each injection site. Similar values would fall near the solid line (slope $=1$ ), whereas uncorrelated values would tend to lie farther from the solid line. Our sample shows that the correlation (coefficient $r=0.96)$ holds across all orientation values $\left(0-180^{\circ}\right)$.

razor-sharp, nor is the representation of visual space in cortex (Hubel and Wiesel, 1974; Dow et al., 1981).

The extent of the projections with respect to receptive field size sheds light on the issue of center-surround effects in primates (Allman et al., 1985). Several studies have examined how surrounding contexts affect the responses within a receptive field (Knierim and Van Essen, 1992; Sillito et al., 1995; Zipser et al.,1996; Levitt and Lund, 1997; Nothdurft et al., 1999), in some cases showing axial specificity (Kapadia et al., 1995). Some authors have proposed that lateral projections supply the modulatory influences. One question is whether the projections reach far enough to provide these surround effects or whether a polysynaptic chain needs to be evoked. Our measurements suggest a 1-2 $\mathrm{mm}$ limit on the area over which any monosynaptic influence might be expected. In squirrel monkeys, this is equivalent to no more than $0.5^{\circ}$ beyond the borders of the receptive field (at $1^{\circ}$ eccentricity), similar to what has been found in macaques (Yoshioka et al., 1996). The range of influence is therefore no more than one receptive field diameter on any side, creating an "integrating field" that is $\sim 10$ times the area of the receptive field, comparable with what has been reported in the cat (Gilbert et al., 1996). Such an integrative field has been demonstrated directly in cat V1, where depolarizing inputs to a neuron were recorded from a region approximately three times the diameter of the minimum response field (Bringuier et al., 1999). Surround modulation at longer distances therefore probably requires the action of polysynaptic relays or feedback from extrastriate areas.

Bosking et al. (1997) suggested that projections that align with retinotopic axes may produce the facilitation with axial specificity needed to integrate contours. This idea cannot be readily applied to primates because of significant differences between tree shrew and monkey V1. (1) Projections in the tree shrew span an equivalent of $20^{\circ}$ of the visual field, whereas those in the primate cover less than a tenth of that amount. (2) Neurons in tree shrew V1 show continuously increasing responses to line stimuli, extending far beyond the limits of the receptive field (Bosking and Fitzpatrick, 1995), whereas complex cell responses in primates either plateau or show distinct end-stopping to long stimuli (Hubel and 
A
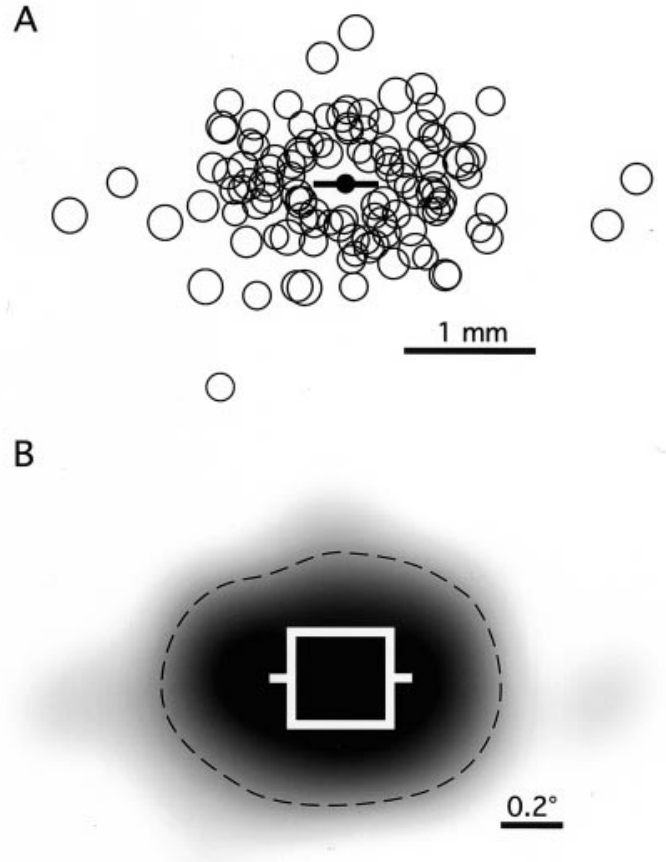

Figure 9. Squirrel monkey lateral projections span approximately three times the average receptive field diameter. $A$, The patch systems of 10 injection sites from squirrel monkeys are aligned by their preferred orientation and superimposed. The heavy line is proportional to the vector average computed for the entire group of patches. B, A Gaussiansmoothed depiction of the patches in $A$ is shown. The white rectangle represents the average receptive field size for these injection sites $(0.3 \times$ $\left.0.32^{\circ}\right)$. The dashed outline is a visual aid, encompassing all values darker than a $10 \%$ gray level.

Wiesel, 1968; Schiller et al., 1976b). Instead, the axial alignment of the projections in primates may underlie length summation within the receptive field rather than beyond it. This is commonly observed in macaques (Hubel and Wiesel, 1968; Schiller et al., 1976a; Kapadia et al., 1999) and in cats (Hubel and Wiesel, 1962; Gilbert, 1977; Rose, 1977; Henry et al., 1978; DeAngelis et al., 1994). The average spacing of iso-orientation columns (Sincich, 1999) and the average receptive field size we recorded suggest that there is significant overlap of receptive fields with the same orientation preference. If an optimal stimulus is presented over one receptive field, it will also impinge on a number of overlapping receptive fields preferring the same stimulus. Because patchy projections are reciprocally connected by glutamatergic synapses (Rockland and Lund, 1983; LeVay, 1988; Kisvárday and Eysel, 1992), this suggests that some recurrent excitation will bolster responses to line stimuli. Specifically, responses to a short line centered in the receptive field will increase as the line is made longer, stimulating more of the overlapping receptive fields as it lengthens. In support of this mechanism for length summation is the finding that orientation tuning sharpens with increasing line length within the receptive field [Schiller et al. (1976b), their Fig. 7], although other mechanisms could be responsible.

All of the layers that house striking patterns of lateral projections are also home to the first neurons in the primate visual pathway that respond specifically to oriented stimuli. Neurons in layer 3 lie one synapse downstream from the geniculate-receptive layers $4 \mathrm{C} \beta$ and $4 \mathrm{~A}$, which have few cells with orientation selectivity (Hubel and Wiesel, 1968; Dow, 1974; Bullier and Henry, 1980; Blasdel and Fitzpatrick, 1984; Anderson et al., 1993;
Ringach et al., 1997). Layer 4B, the intralaminar projections of which form the stria of Gennari, is also one synapse removed from its geniculate input in layer $4 \mathrm{C} \alpha$ (Fitzpatrick et al., 1985; Yabuta and Callaway, 1998a). Layer 4B has a system of axon terminal patches lying in tight register with that in the upper layers (Fig. 3) (Rockland and Lund, 1983). This suggests that the layer 4B patch system might also show an elongated pattern that lines up with orientation preferences. To see whether this is true, experiments with injections targeted to layer 4B would have to be done.

Because this arrangement is present in the same layers in which orientation selectivity is so pronounced, our result lends support to the idea that the patchy projections are in a position to bolster responses to oriented edges. These projections are laid out in an equivalent way to the proposal of Hubel and Wiesel for generating orientation-tuned cells in layer 4 of the cat. In their model, the distribution of geniculate inputs is spatially similar to the stimulus (Hubel and Wiesel, 1962). The lateral projections in New World monkey V1 seem to follow this rule, independent of ocular dominance and other possible anisotropies. They will add to any convergent input arriving from layer 4C. By taking advantage of the retinotopy preserved in the cortical sheet, they appear to retrieve spatial information required for an early step in form perception.

\section{REFERENCES}

Adams JC (1992) Biotin amplification of biotin and horseradish peroxidase signals in histochemical stains. J Histochem Cytochem 40:1457-1463.

Allman JM, Kaas JH (1971) Representation of the visual field in striate and adjoining cortex of the owl monkey (Aotus trivirgatus). Brain Res 35:89-106.

Allman JM, Miezin F, McGuinness E (1985) Stimulus specific responses from beyond the classical receptive field: neurophysiological mechanisms for local-global comparisons in visual neurons. Annu Rev Neurosci 8:407-430.

Amir Y, Harel M, Malach R (1993) Cortical hierarchy reflected in the organization of intrinsic connections in macaque monkey visual cortex. J Comp Neurol 334:19-46.

Anderson JC, Martin KAC, Whitteridge D (1993) Form, function, and intracortical projections of neurons in the striate cortex of the monkey Macacus nemestrinus. Cereb Cortex 3:412-420.

Barlow HB, Blakemore C, Pettigrew JD (1967) The neural mechanism of binocular depth discrimination. J Physiol (Lond) 193:327-342.

Blasdel GG (1992) Differential imaging of ocular dominance and orientation selectivity in monkey striate cortex. J Neurosci 12:3115-3138.

Blasdel GG, Campbell DC (2001) Functional retinotopy of monkey visual cortex. J Neurosci, in press.

Blasdel GG, Fitzpatrick D (1984) Physiological organization of layer 4 in macaque striate cortex. J Neurosci 4:880-895.

Bosking WH, Fitzpatrick D (1995) Physiological correlates of anisotropy in horizontal connections: length summation properties of neurons in layers 2 and 3 of tree shrew striate cortex. Soc Neurosci Abstr 21:1751.

Bosking WH, Zhang Y, Schofield B, Fitzpatrick D (1997) Orientation selectivity and the arrangement of horizontal connections in tree shrew striate cortex. J Neurosci 17:2112-2127.

Bringuier V, Chavane F, Glaeser L, Frégnac Y (1999) Horizontal propagation of visual activity in the synaptic integration field of area 17. Science 283:695-699.

Bullier J, Henry GH (1980) Ordinal position and afferent input of neurons in monkey striate cortex. J Comp Neurol 193:913-935.

Campbell DC, Blasdel GG (1995) Optical measurement of cortical magnification factors in New and Old World primates. Soc Neurosci Abstr 21:771.

Cowey A (1964) Projection of the retina on to striate and prestriate cortex in the squirrel monkey Saimiri sciureus. J Neurophysiol 27:366-393.

DeAngelis GC, Freeman RD, Ohzawa I (1994) Length and width tuning of neurons in the cat's primary visual cortex. J Neurophysiol 71:347-374.

DeFelipe J, Fariñas I (1992) The pyramidal neuron of the cerebral cortex: morphological and chemical characteristics of the synaptic inputs. Prog Neurobiol 39:563-607. 
Dow BM (1974) Functional classes of cells and their laminar distribution in monkey visual cortex. J Neurophysiol 37:927-946.

Dow BM, Synder AZ, Vautin RG, Bauer R (1981) Magnification factor and receptive field size in foveal striate cortex of the monkey. Exp Brain Res 44:213-228.

Dow BM, Vautin RG, Bauer R (1985) The mapping of visual space onto foveal striate cortex in the macaque monkey. J Neurosci 5:890-902.

Ferster D, Miller KD (2000) Neural mechanisms of orientation selectivity in the visual cortex. Annu Rev Neurosci 23:441-471.

Fitzpatrick D, Lund JS, Blasdel GG (1985) Intrinsic connections of macaque striate cortex: afferent and efferent connections of lamina 4C. J Neurosci 5:3329-3349.

Gilbert CD (1977) Laminar differences in receptive field properties of cells in cat primary visual cortex. J Physiol (Lond) 268:391-421.

Gilbert CD, Wiesel TN (1989) Columnar specificity of intrinsic horizontal and corticocortical connections in cat visual cortex. J Neurosci 9:2432-2442.

Gilbert CD, Das A, Ito M, Kapadia M, Westheimer G (1996) Spatial integration and cortical dynamics. Proc Natl Acad Sci USA 93:615-622.

Hendrickson AE, Wilson JR, Ogren MP (1978) The neuroanatomical organization of pathways between the dorsal lateral geniculate nucleus and visual cortex in Old World and New World primates. J Comp Neurol 182:123-136.

Henry GH, Goodwin AW, Bishop PO (1978) Spatial summation of responses in receptive fields of single cells in cat striate cortex. Exp Brain Res 32:245-266.

Horikawa K, Armstrong WE (1988) A versatile means of intracellular labeling: injection of biocytin and its detection with avidin conjugates. J Neurosci Methods 25:1-11.

Horton JC, Hocking DR (1996) Anatomical demonstration of ocular dominance columns in striate cortex of the squirrel monkey. J Neurosci $16: 5510-5522$.

Hubel DH, Wiesel TN (1962) Receptive fields, binocular interaction and functional architecture in the cat's visual cortex. J Physiol (Lond) 160:106-154

Hubel DH, Wiesel TN (1968) Receptive fields and functional architecture of monkey striate cortex. J Physiol (Lond) 195:215-243.

Hubel DH, Wiesel TN (1974) Uniformity of monkey striate cortex: a parallel relationship between field size, scatter, and magnification factor. J Comp Neurol 158:295-306.

Kaas JH, Lin C-S, Casagrande VA (1976) The relay of ipsilateral and contralateral retinal input from the lateral geniculate nucleus to striate cortex in the owl monkey: a transneuronal transport study. Brain Res $106: 371-378$

Kapadia MK, Ito M, Gilbert CD, Westheimer G (1995) Improvement in visual sensitivity by changes in local context: parallel studies in human observers and in V1 of alert monkeys. Neuron 15:843-856.

Kapadia MK, Westheimer G, Gilbert CD (1999) Dynamics of spatial summation in primary visual cortex of alert monkeys. Proc Natl Acad Sci USA 96:12073-12078.

Kisvárday ZF, Eysel UT (1992) Cellular organization of reciprocal patchy networks in layer III of cat visual cortex (area 17). Neuroscience 46:275-286.

Knierim JJ, Van Essen DC (1992) Neuronal responses to static texture patterns in area V1 of the alert macaque monkey. J Neurophysiol 67:961-980.

LeVay S (1988) Patchy intrinsic projections in visual cortex, area 18, of the cat: morphological and immunocytochemical evidence for an excitatory function. J Comp Neurol 269:265-274.

Levitt JB, Lund JS (1997) Contrast dependence of contextual effects in primate visual cortex. Nature 387:73-76.

Livingstone MS, Hubel DH (1984) Specificity of intrinsic connections in primate primary visual cortex. J Neurosci 4:2830-2835.

Lund JS, Wu CQ (1997) Local circuit neurons of macaque monkey striate cortex. IV. Neurons of laminae 1-3A. J Comp Neurol 384:109-126.

Lund JS, Yoshioka T (1991) Local circuit neurons of macaque monkey striate cortex. III. Neurons of laminae 4B, 4A, and 3B. J Comp Neurol 311:234-259.

Malach R, Amir Y, Harel M, Grinvald A (1993) Relationship between intrinsic connections and functional architecture revealed by optical imaging and in vivo targeted biocytin injections in primate striate cortex. Proc Natl Acad Sci USA 90:10469-10473.

McGuire BA, Gilbert CD, Rivlin P, Wiesel TN (1991) Targets of horizontal connections in macaque primary visual cortex. J Comp Neurol 305:370-392

McLoughlin NP, Blasdel GG (1998) Wavelength-dependent differences between optically determined functional maps from macaque striate cortex. NeuroImage 7:326-336.

Nothdurft H-C, Gallant JL, Van Essen DC (1999) Response modulation by texture surround in primate area V1: correlates of "popout" under anesthesia. Vis Neurosci 16:15-34.

O'Keefe LP, Levitt JB, Kiper DC, Shapley RM, Movshon JA (1998) Functional organization of owl monkey lateral geniculate nucleus and visual cortex. J Neurophysiol 80:594-609.

Poggio GF (1972) Spatial properties of neurons in striate cortex of unanesthetized macaque monkey. Invest Ophthalmol 11:368-377.

Ringach DL, Hawken MJ, Shapley R (1997) Dynamics of orientation tuning in macaque primary visual cortex. Nature 387:281-284.

Rockland KS, Lund JS (1983) Intrinsic laminar lattice connections in primate visual cortex. J Comp Neurol 216:303-318.

Rose DJ (1977) Responses of single units in cat visual cortex to moving bars of light as a function of bar length. J Physiol (Lond) 271:1-23.

Schiller PH, Finlay BL, Volman SF (1976a) Quantitative studies of single-cell properties in monkey striate cortex. I. Spatiotemporal organization of receptive fields. J Neurophysiol 39:1288-1319.

Schiller PH, Finlay BL, Volman SF (1976b) Quantitative studies of single-cell properties in monkey striate cortex. II. Orientation specificity and ocular dominance. J Neurophysiol 39:1320-1333.

Schmidt KE, Goebel R, Löwel S, Singer W (1997) The perceptual grouping criterion of colinearity is reflected by anisotropies of connections in the primary visual cortex. Eur J Neurosci 9:1083-1089.

Sillito AM, Grieve KL, Jones HE, Cudeiro J, Davis J (1995) Visual cortical mechanisms detecting focal orientation discontinuities. Nature 378:492-496.

Sincich LC (1999) Local circuits in primary visual cortex. PhD thesis, Harvard University.

Skottun BC, De Valois RL, Grosof DH, Movshon JA, Albrecht DG, Bonds AB (1991) Classifying simple and complex cells on the basis of response modulation. Vision Res 31:1079-1086.

Snodderly DM, Gur M (1995) Organization of striate cortex of alert, trained monkeys (Macaca fascicularis): ongoing activity, stimulus selectivity, and widths of receptive field activating regions. J Neurophysiol $74: 2100-2125$.

Tootell RBH, Switkes E, Silverman MS, Hamilton SL (1988) Functional anatomy of macaque striate cortex. II. Retinotopic organization. J Neurosci 8:1531-1568.

Van Essen DC, Newsome WT, Maunsell JHR (1984) The visual field representation in striate cortex of the macaque monkey: asymmetries, anisotropies, and individual variability. Vision Res 24:429-448.

Weliky M, Kandler K, Fitzpatrick D, Katz LC (1995) Patterns of excitation and inhibition evoked by horizontal connections in visual cortex share a common relationship to orientation columns. Neuron 15:541-552.

Wong-Riley M (1979) Changes in the visual system of monocularly sutured or enucleated cats demonstrable with cytochrome oxidase histochemistry. Brain Res 171:11-28.

Yabuta NH, Callaway EM (1998a) Functional streams and local connections of layer $4 \mathrm{C}$ neurons in primary visual cortex of the macaque monkey. J Neurosci 18:9489-9499.

Yabuta NH, Callaway EM (1998b) Cytochrome-oxidase blobs and intrinsic horizontal connections of layer $2 / 3$ pyramidal neurons in primate V1. Vis Neurosci 15:1007-1027.

Yoshioka T, Blasdel GG, Levitt JB, Lund JS (1996) Relation between patterns of intrinsic lateral connectivity, ocular dominance, and cytochrome oxidase-reactive regions in macaque monkey striate cortex. Cereb Cortex 6:297-310.

Zipser K, Lamme VAF, Schiller PH (1996) Contextual modulation in primary visual cortex. J Neurosci 16:7376-7389. 